\title{
„Verbleib“ von in Deutschland gewählten Europa-Abgeordneten
}

\author{
Peter Rütters
}

Auch heute noch wird das Europäische Parlament (EP) nicht selten - und kontrafaktisch - als randständige Institution eingeordnet: arm an Einfluss und dürftig an sachlicher und personeller Gestaltungskraft. ${ }^{1}$ Es sollte daher nicht überraschen, dass seine Abgeordneten vom Wahlbürger und weiten Teilen der Öffentlichkeit so gut wie nicht wahrgenommen werden. ${ }^{2}$ Ein Mandat im Europäischen Parlament scheint aus dieser Perspektive einen geringen Status zu haben und wenig Chancen für einen Aufstieg in exekutive Funktionen (Mitglied oder Präsident der Kommission der EU) zu bieten. Es mag dann auch nicht verwundern, dass sich Vorstellungen halten, dass Mandate im EP zur Versorgung von Politikern dienen oder als Abfindung zum Ende einer langen politischen Karriere genutzt werden. ${ }^{3}$ Das böse Wort: „Hast du einen Opa, dann schick ihn nach Europa“ scheint längst nicht in Vergessenheit geraten zu sein.

Darin drückt sich eine Distanz zwischen Repräsentanten und Repräsentierten aus. Mit derzeit 99 Abgeordneten kommen auf jeden in Deutschland gewählten Parlamentarier im Durchschnitt ungefähr 820.000 Einwohner und etwa 630.000 Wahlberechtigte. Diese Relationen erlauben es dem einzelnen Abgeordneten nicht, sich wähler- und bevölkerungsnah zu präsentieren. Für die Nominierung als Kandidat für die Europawahl und für die Platzierung auf (starren) Wahllisten der Parteien, aber auch für eine bürgerbezogene Responsivität steht für den Abgeordneten nicht die Beziehung zum Wähler im Vordergrund, sondern von ausschlaggebender Bedeutung sind seine Position und Akzeptanz in seiner Partei und in den für die Nominierung zuständigen Parteigremien. ${ }^{4}$

In der Politikwissenschaft besteht breiter Konsens darüber, dass Parlamenten wie dem Bundestag, den Landtagen und inzwischen auch dem EP eine Schlüsselposition in repräsentativ-demokratischen politischen Systemen zukommt: als Legislative, durch Wahlfunktionen (Bundeskanzler, Ministerpräsidenten, Wahl / Bestätigung des Präsidenten und der Mitglieder der Kommission der EU) und durch die Kontrolle exekutiven Handels (der Regierung, der Kommission der EU). 5 Die Abgeordneten in den Parlamenten sind die personellen und

1 Vgl. Oskar Niedermayer, Das Europäische Parlament in der öffentlichen Meinung - bekannt, aber wenig relevant, in: integration, 32. Jg. (2009), H. 3, S. $231-245$.

2 Vgl. ders., Die Wahl zum Europäischen Parlament vom 7. Juni 2009 in Deutschland: SPD-Debakel im Vorfeld der Bundestagswahl, in: ZParl, 40. Jg. (2009), H. 4, S. 711 - 731, S. 721, Anmerkung 28.

3 Vgl. auch Jürgen Mittag, Wegmarke für die Parlamentarisierung der Europäischen Union: Die finanziellen Neuregelungen des europäischen Abgeordnetenhauses, in: ZParl, 37. Jg. (2006), H. 4, S. 713 - 728, S. 713, der auf die lange Zeit vorherrschende Wahrnehmung des Europäischen Parlaments als „Versorgungsposten für verdiente oder, wegzulobende' nationale Politiker“ hinweist.

4 Vgl. Werner J. Patzelt, Abgeordnete und ihr Beruf. Interviews - Umfragen - Analysen, Berlin 1995, S. 235 f., S. 239 ff., S. 245 f.; ders., Abgeordnete und Repräsentation. Amtsverständnis und Wahlkreisarbeit, Passau 1993, S. 256 - 261, S. 382 - 390; ferner Benjamin Höhne, Rekrutierung von Abgeordneten des Europäischen Parlaments. Organisation, Akteure und Entscheidungen in Parteien, Opladen 2013.

5 Vgl. für viele Stefan Marschall, Parlamentarismus. Eine Einführung, Baden-Baden 2005, S. 133 -193 . 
individuellen Träger der politischen Entscheidungen, die Repräsentanten der (Wahl-)Bevölkerung und eines allgemeinen Wählerwillens, und sie sind mehr oder weniger unmittelbar gegenüber der Bevölkerung verantwortlich (Responsivität). Eine zeitliche Befristung und das Ausscheiden aus dem Parlament sind inhärente Elemente des Abgeordnetenmandats in einer repräsentativen Demokratie. Der Eintritt neuer Abgeordneter wie auch das Ausscheiden bisheriger Mandatsträger sind ebenso selbstverständliche Aspekte des Parlamentsalltags wie notwendige Elemente des Parlamentarismus in einer repräsentativen Demokratie, in der mittels Wahlen politische Repräsentanten für eine begrenzte Zeit legitimiert und periodisch einer Art Erfolgskontrolle unterzogen werden. Die Funktionsanforderungen von Parlamenten verlangen aber auch, dass eine nicht eindeutig festzulegende Anzahl von Abgeordneten länger als nur eine Wahlperiode einem Parlament angehört.

Die Sozialstruktur von Abgeordneten, ihre politischen Karrierewege bis zur Erlangung des Mandats, ihre Stellung und ihr Aufstieg im Parlamentssystem bilden einen traditionellen Untersuchungsgegenstand der politischen Soziologie und der Politikwissenschaft. 6 Obwohl dieses Forschungsfeld kontinuierlich bearbeitet wird, zeigt sich eine Fokussierung auf das nationale Parlament, in der Bundesrepublik seit 1949 auf den Bundestag, während bereits die Landesparlamente und ihre Abgeordneten eher beiläufig und diskontinuierlich zum Gegenstand ähnlicher Forschungsvorhaben erhoben wurden. ${ }^{7}$ Eine noch geringere Beachtung hat, abgesehen von einer kleinen Forschungseuphorie nach der ersten Direktwahl, das Europäische Parlament erfahren. ${ }^{8}$ Den deutschen Europa-Parlamentariern wurde, wiederum

6 Zur umfangreichen Literatur vgl. unter anderem Peter Schindler, Datenhandbuch zur Geschichte des Deutschen Bundestages 1949 bis 1999, Bd. 1, Baden-Baden 1999, S. 554 - 866; Michael F. Feldkamp, Datenhandbuch zur Geschichte des Deutschen Bundestages 1990 bis 2010, BadenBaden 2011, S. 249 - 407; Adalbert Hess, Sozialstruktur des 13. Bundestages. Berufliche und fachliche Entwicklungslinien, in: ZParl, 26. Jg. (1995), H. 4, S. 567 - 585; Franziska Deutsch I Suzanne S. Schüttemeyer, Die Berufsstruktur des Deutschen Bundestages: 14. und 15. Wahlperiode, in: ZParl, 34. Jg. (2003), H. 1, S. 21 - 32; Melanie Kintz, Die Berufsstruktur der Abgeordneten des 17. Deutschen Bundestages, in: ZParl, 41. Jg. (2010), H. 3, S. 491 - 503; ferner die Literaturangaben in: Peter Schindler, ebenda, S. 680 f.; Hartmut Klatt, Das Sozialprofil des Deutschen Bundestages, 1949 - 1976: Das parlamentarische Personal kommt aus der Mittelschicht, in: Gegenwartskunde, 28. Jg. (1979), H. 1, S. 65 - 80; Emil-Peter Müller, Der Bundestag ist gebildeter geworden. Zur Entwicklung des Bildungsstandes der Bundestagsabgeordneten seit 1949, in: ZParl, 19. Jg. (1988), H. 2, S. 200 - 219; ferner Maria Kreiner, Amt auf Zeit. Eine Verbleibsstudie über ehemalige Bundestagsabgeordnete, Baden-Baden 2006, S. 16 - 19, die in Anmerkung 14 bis 18 aufgelistete Literatur; auch Werner J. Patzelt, Abgeordnete und ihr Beruf, a.a.O. (Fn. 4).

7 Vgl. die einzelnen Länderartikel in: Siegfried Mielke / Werner Reutter (Hrsg.), Landesparlamentarismus. Geschichte - Struktur - Funktionen, Wiesbaden 2012; Hans-Ulrich Derlien / Stefan Lock, Eine neue politische Elite? Rekrutierung und Karrieren der Abgeordneten in den fünf neuen Landtagen, in: ZParl, 25. Jg. (1994), H. 1, S. 61 - 94.

8 Vgl. Emil J. Kirchner, The European Parliament: Performance and Prospects, Aldershot 1984; Eva M. Thöne, Das direkt gewählte Europäische Parlament. Ein Beitrag zur Abgeordnetensoziologie, in: ZParl, 13. Jg. (1982), H. 2, S. 149 - 180; Richard Corbett / Francis Jacobs / Michael Shackleton, The European Parliament, London 2011, S. 51 - 64, einschließlich eines Abschnitts (S. 59 ff.) über politische Positionen nach dem Ausscheiden aus dem Europäischen Parlament, sowie die vorangehenden sieben Ausgaben seit 1990; ferner Eberhard Grabitz / Otto Schmuck / Sabine Steppat / Wolfgang Wessels, Direktwahl und Demokratisierung - Eine Funktionsbilanz des Europäischen Parlaments nach der ersten Wahlperiode, Bonn 1988; die zuletzt angeführte Studie verdeutlicht indes, dass eine Bilanz des Europäischen Parlaments auf eine differenzierte Sozialstruktur-Analyse der Abgeordneten meint verzichten zu können. Dennoch sollte es irritieren, dass ein Sammelband, 
nach einer begrenzten Anfangsaufmerksamkeit, eine noch geringere wissenschaftliche Beachtung entgegengebracht. ${ }^{9}$ Den Gründen für dieses rasch abflauende und alles in allem mäßige wissenschaftliche Interesse soll aber an dieser Stelle nicht weiter nachgegangen werden. ${ }^{10}$

Es wird nun nicht überraschen, dass das Ausscheiden von (deutschen) Abgeordneten aus dem EP, deren Verbleib und ihre weiteren politischen und gesellschaftspolitischen Karrierewege und Ambitionen bislang wissenschaftlich nicht untersucht wurden, denn schon für die nationale Ebene - den Bundestag und die Landesparlamente - können Verbleib-Studien als Rarität gelten. ${ }^{11}$ Doch sollte dies als ein irritierender Befund angesehen werden, weil nicht davon auszugehen ist, dass das Mandat immer den Höhe- und Endpunkt einer politischen und beruflichen Karriere darstellt, auch nicht für Europa-Abgeordnete. Das Ausscheiden aus dem Parlament ist zudem kein randständiges Phänomen, das nach jeder Wahlperiode nur

der thematisch 30 Jahre Direktwahl des Europäischen Parlaments fokussiert, abgesehen von wenigen Hinweisen in der Einleitung des Herausgebers von den Abgeordneten des Europäischen Parlaments eigentlich keine Notiz nimmt, vgl. Jürgen Mittag (Hrsg.), 30 Jahre Direktwahlen zum Europäischen Parlament (1979 - 2009). Europawahlen und EP in der Analyse, Baden-Baden 2011; ähnlich bereits Andreas Maurer / Dietmar Nickel (Hrsg.), Das Europäische Parlament. Supranationalität, Repräsentation und Legitimation, Baden-Baden 2005.

9 Vgl. Karlheinz Reif/ Hermann Schmitt / Klaus Schubert, Wer sind und was wollen die Deutschen im Europäischen Parlament? Sozialprofil, politischer Rückhalt und Zielvorstellungen der deutschen Kandidaten zum Europäischen Parlament, in: ZParl, 10. Jg. (1979), H. 3, S. 332 - 354; RudolfHrbek / Carl-Christoph Schweitzer, Die deutschen Europa-Parlamentarier. Ergebnisse einer Befragung der deutschen Mitglieder des Europäischen Parlaments, in: APuZ, 39. Jg. (1989), B 3, S. 3 - 18; für die Zeit vor der Direktwahl des EP: Peter Reichel, Bundestagsabgeordnete in europäischen Parlamenten. Zur Soziologie des europäischen Parlamentariers, Hamburg 1974.

10 Verwiesen sei auf die anfangs bestehende Kompetenzarmut des Europäischen Parlaments, die relativ geringe Anzahl der deutschen EP-Abgeordneten (81, seit 1994: 99) und den Nimbus des EP, politisch und parlamentarisch von geringer Bedeutung zu sein, so dass hier langgediente Parlamentarier ihre politische Karriere ausklingen lassen können oder Politiker mit nationalen Ambitionen für eine begrenzte Zeit „geparkt“ werden, damit sie den Kontakt zum politischen Leben nicht verlieren und gleichzeitig alimentiert sind. Im Vergleich zu den Abgeordneten fand das EP als Institution und seine Funktion in der Europäischen Gemeinschaft / Europäischen Union stetiges wissenschaftliches Interesse.

11 Für den Bundestag liegt bislang nur die Arbeit von Maria Kreiner, a.a.O. (Fn. 6) vor, die auf der Grundlage von 38 leitfadengestützten Interviews eine qualitative Verbleibsstudie vorgelegt hat, die Abgeordnete der 12. und 13. Wahlperiode erfasst, die mit dem jeweiligen Ende der Wahlperiode 1994 beziehungsweise 1998 ausgeschieden waren. Ein Schwerpunkt dieser Untersuchung liegt auf der sozialen und beruflichen Absicherung der ausgeschiedenen Parlamentarier. Vgl. auch dies., Amt auf Zeit. Eine explorative Studie zum beruflichen und politischen Verbleib ehemaliger Bundestagsabgeordneter, in: ZParl, 38. Jg. (2007), H. 2, S. 261 - 276. Eine quantitativ umfangreichere Untersuchung über ausgeschiedene Parlamentarier stellt die Studie von Michael Edinger / Bertram Schwarz, Leben nach dem Mandat. Eine Studie zu ehemaligen Abgeordneten, Jena 2009, dar, die im Rahmen des Sonderforschungsbereichs 580 „Gesellschaftliche Entwicklungen nach dem Systemumbruch. Diskontinuität, Tradition und Strukturbildung “ entstanden ist. Diese Studie wertete biographische Daten von ausgeschiedenen Abgeordneten des Bundestages und von Landesparlamenten aus, die zwischen 1991 und 2002 und 2003 bis 2007 aus den Parlamenten ausgeschiedenen waren, und vertiefte die Informationsbasis durch fragebogengestützte Telefoninterviews. Die Auswertung, die unter anderem die Gründe für die Mandatsbeendigung, die Ausübung von nachparlamentarischen Ämtern und beruflichen Aktivitäten sowie das fortgesetzte politische Engagement erfasste, verzichtet auf eine zeitliche Differenzierung der Abgeordneten und unterscheidet nur (vergleichend) zwischen Abgeordneten des Bundestages und von Landesparlamenten. 
wenige Abgeordnete trifft. Beispielsweise verlassen im Durchschnitt etwa 30 Prozent der Parlamentarier mit dem Ende einer Wahlperiode den Bundestag. ${ }^{12}$ Aus unterschiedlichen Gründen variiert der Anteil der (deutschen) Abgeordneten, die mit dem Ende einer Wahlperiode aus dem EP ausgeschieden sind, seit Einführung der Direktwahl zwischen etwa 30 und 50 Prozent (vgl. Tabelle 1).

Die Frage nach den Gründen für das nicht immer freiwillige Ausscheiden ${ }^{13}$ aus dem EP, nach der weiteren politischen Perspektive und dem folgenden politischen und gesellschaftspolitischen Engagement dieser Abgeordneten soll für zwei Gruppen untersucht werden: (1) Zum einen für jene, die im Laufe einer Wahlperiode das EP verließen, für die - abgesehen von den während der Wahlperiode Verstorbenen oder krankheitsbedingt Ausgeschiedenen - ein freiwilliger und selbstbestimmter Verzicht auf das Parlamentsmandat angenommen werden kann. Für diese zahlenmäßig überschaubare Gruppe können hier die Abgeordneten aller Wahlperioden des EP berücksichtigt werden; (2) zum anderen sollen jene Abgeordneten untersucht werden, die mit dem Ende der Wahlperiode das EP verließen oder verlassen mussten.

Da diese Gruppe zahlenmäßig ungleich größer ist, beschränkt sich diese als explorativ verstandene Untersuchung ${ }^{14}$ auf die Abgeordneten, die mit dem Ende der 6. Wahlperiode aus dem Europäischen Parlament ausgeschieden sind. ${ }^{15}$

\section{Ausscheiden während der Wahlperiode}

Die überschaubare Gruppe von Abgeordneten, deren Mandat während der Wahlperiode endete $^{16}$, unterscheidet sich von jenen, die mit dem Ende einer Wahlperiode nicht mehr

12 Vgl. Michael F. Feldkamp, a.a.O. (Fn. 6), S. 265.

13 Die Untersuchung zum Ausscheiden von Abgeordneten aus dem Europäischen Parlament bezieht sich nur auf die in der Bundesrepublik Deutschland von 1979 bis 2009 gewählten Parlamentarier (von 1979 bis 1989 in der „alten“ Bundesrepublik, zuzüglich drei vom Berliner Abgeordnetenhaus delegierte Abgeordnete; seit der Wahl von 1994 auf die Abgeordneten, die in der Bundesrepublik Deutschland einschließlich der neuen Bundesländer und Berlins gewählt wurden). Wenn im folgenden Text und in den Tabellen mit Bezug auf das Europäische Parlament verkürzt von Abgeordneten / Parlamentariern gesprochen wird, ist damit immer nur diese Gruppe der Mitglieder des Europäischen Parlaments gemeint.

14 Explorativ in dem Sinne als am Beispiel der EP-Abgeordneten auf das - trotz der beiden erwähnten Studien - bislang immer noch geringe politik- und sozialwissenschaftliche Interesse an Verbleibstudien über ausgeschiedene Parlamentarier hingewiesen werden soll.

15 Diese Eingrenzung hat forschungspragmatische Gründe: Informationen zum Ausscheiden und Verbleib der Abgeordneten wurden vor allem über das Internet erhoben (Homepages ehemaliger MdEP und sonstige Informationen im Internet, die mit einer einfachen, namensbezogenen Suchrecherche zu finden waren). Ausreichende Ergebnisse konnten daher nur für zeitnah ausgeschiedene Abgeordnete erwartet werden. Hilfreiche Informationen zu einzelnen ausgeschiedenen Abgeordneten bot darüber hinaus noch die bereits erwähnte Studie von Benjamin Höhne, a.a.O. (Fn. 4), die die Kandidatenaufstellung deutscher Parteien für die Europawahl im Jahr 2009 in den Mittelpunkt stellte. Für eine Untersuchung, die alle Abgeordneten seit der 1. Wahlperiode berücksichtigen würde, wären erheblich aufwendigere und auf andere Quellen gestützte Recherchen erforderlich.

16 Vgl. hierzu auch den Abschnitt „Replacement of MEPs“ über die in den ersten vier Jahren der ersten Wahlperiode ausgeschiedenen EP-Abgeordneten in: Emil J. Kirchner, a.a.O. (Fn. 8), S. 6-12. 
Tabelle 1: Dauer der Mitgliedschaft im Europäischen Parlament (1979 bis 2009, Beginn der Wahlperiode, in Prozent)

\begin{tabular}{|c|c|c|c|c|c|c|c|c|}
\hline & $\begin{array}{c}\text { vor/bis } \\
1979\end{array}$ & $\begin{array}{c}\text { 1. WP } \\
(1979 \text { bis } \\
1984)\end{array}$ & $\begin{array}{l}\text { 2. WP } \\
(1984 \text { bis } \\
1989)\end{array}$ & $\begin{array}{l}\text { 3. WP } \\
\text { (1989 bis } \\
1994)\end{array}$ & $\begin{array}{c}\text { 4. WP } \\
(1994 \text { bis } \\
1999)\end{array}$ & $\begin{array}{c}5 . \mathrm{WP} \\
(1999 \text { bis } \\
2004)\end{array}$ & $\begin{array}{c}\text { 6. WP } \\
(2004 \text { bis } \\
2009)\end{array}$ & $\begin{array}{l}\text { 7.WP } \\
\text { (2009 bis } \\
2014)\end{array}$ \\
\hline vor/bis 1979 & $(100,0)$ & 19,8 & 12,3 & 3,7 & 1,0 & - & - & - \\
\hline 1. WP (1979 bis 1984$)$ & & 80,2 & $56,8^{a}$ & $33,3^{\mathrm{b}}$ & $14,1 \mathrm{e}$ & $9,1^{\mathrm{i}}$ & $3,0^{\mathrm{n}}$ & $2,0^{\mathrm{t}}$ \\
\hline 2. WP (1984 bis 1989) & & - & 30,9 & $14,8^{c}$ & $7,1^{f}$ & $4,0^{\mathrm{k}}$ & $4,0^{\circ}$ & - \\
\hline 3. WP (1989 bis 1994$)$ & & - & & $48,1^{\mathrm{d}}$ & $24,2 \mathrm{~g}$ & $18,2^{1}$ & $10,1 \mathrm{p}$ & 5,1 \\
\hline 4. WP (1994 bis 1999) & & - & & & $53,5^{\mathrm{h}}$ & $39,4^{m}$ & 30,39 & $19,2^{u}$ \\
\hline 5. WP (1999 bis 2004) & & - & & & & 29,3 & $20,2^{\mathrm{r}}$ & $10,1^{\mathrm{v}}$ \\
\hline 6. WP (2004 bis 2009) & & - & & & & & $32,3^{\mathrm{s}}$ & $22,2^{\mathrm{w}}$ \\
\hline 7. WP seit 2009 & & - & & & & & & $41,4^{x}$ \\
\hline insgesamt (in Prozent) & & 100 & 100 & 100 & 100 & 100 & 100 & 100 \\
\hline insgesamt (absolut) & (36) & 81 & 81 & 81 & 99 & 99 & 99 & 99 \\
\hline
\end{tabular}

a Davon sieben Abgeordnete als Nachrücker in der 1. Wahlperiode.

b Davon vier Abgeordnete als Nachrücker in der 1. Wahlperiode.

c Davon drei Abgeordnete als Nachrücker in der 2. Wahlperiode.

d Davon drei Abgeordnete diskontinuierlich im EP (Mechthild von Alemann, MdEP 1979 bis 1984; F. W. Graefe zu Baringdorf und Dorothee Piermont diskontinuierlich in EP 1984 bis 1987 wegen Rotationsregelung bei den Grünen).

e Davon ein Abgeordneter (Elmar Brok) als Nachrücker 1980.

f Davon drei Abgeordnete als Nachrücker in der 2. Wahlperiode; ein Abgeordneter (F. W. Graefe zu Baringdorf) diskontinuierlich in EP, seit 3. Wahlperiode erneut und kontinuierlich, siehe d).

g Davon sechs Abgeordnete als Nachrücker in der 3. Wahlperiode (1989 bis 1994); ein Abgeordneter (F. W. Graefe zu Baringdorf) diskontinuierlich, siehe d) und f).

h Davon zwei Abgeordnete diskontinuierlich im EP (Otto Bardong und Undine Bloch von Blottnitz bereits in der 2. Wahlperiode MdEP, aber nicht in der 3. Wahlperiode).

i Davon ein Abgeordneter als Nachrücker (Elmar Brok, 1980).

k Davon eine Abgeordnete als Nachrückerin (Diemut Theatro, 1987).

1 Davon vier Abgeordnete als Nachrücker in der 3. Wahlperiode.

m Davon drei Abgeordnete als Nachrücker in der 4. Wahlperiode.

n Davon ein Abgeordneter als Nachrücker in der 1. Wahlperiode (Elmar Brok, 1980).

o Davon ein Abgeordneter diskontinuierlich im EP in der 2. Wahlperiode (F. W. Graefe zu Baringdorf, 1984 bis 1987), siehe d) und $f$ ).

p Davon zwei Abgeordnete als Nachrücker in der 3. Wahlperiode.

q Davon zwei Abgeordnete als Nachrücker in der 4. Wahlperiode.

r Davon ein Abgeordneter als Nachrücker in der 5. Wahlperiode (Garrelt Duin, 2000).

s Davon ein Abgeordneter diskontinuierlich in EP (Karsten Hoppenstedt, MdEP in 4. Wahlperiode 1994 bis 1999). Daniel Cohn-Bendit, war auch in der 4. Wahlperiode MdEP für die Grünen; in der 5. Wahlperiode 1999 bis 2004 MdEP für Les Verts / Frankreich.

t Davon ein Abgeordneter als Nachrücker in der 1. Wahlperiode (Elmar Brok, MdEP seit 1980).

u Davon zwei Abgeordnete als Nachrücker in der 4. Wahlperiode.

v Davon ein Abgeordneter als Nachrücker in der 5. Wahlperiode.

w Davon zwei Abgeordnete als Nachrücker in der 6. Wahlperiode.

$x$ Davon ein Abgeordneter diskontinuierlich im EP (Bernd Lange war MdEP in der 4. und 5. Wahlperiode 1994 bis 2004). Quelle: Amtliches Handbuch des Europäischen Parlaments 1979 - 1984, 1. Wahlperiode, herausgegeben vom Generalsekretariat des Europäischen Parlaments, Luxemburg 1980 (Loseblatt-Sammlung); Amtliches Handbuch des Europäischen Parlaments 1984, 2. Wahlperiode 1984 - 1989, herausgegeben vom Generalsekretariat des Europäischen Parlaments, Luxemburg 1985 (Stand 1. Dezember 1984); Die Mitglieder des Europäischen Parlaments, 3. Wahlperiode 1989, herausgegeben vom Generalsekretariat des Europäischen Parlaments, Luxemburg 1990 (Stand: April 1990); Die Mitglieder des Europäischen Parlaments, 4. Wahlperiode 1994 - 1999, herausgegeben vom Generalsekretariat des Europäischen Parlaments, Luxemburg 1996 (Stand: Februar 1996); Die Mitglieder des Europäischen Parlaments, 5. Wahlperiode 1999 - 2004, herausgegeben vom Generalsekretariat des Europäischen Parlaments, Luxemburg 2001 (Stand: Juni 2001); Klaus Löffler (Hrsg.), Leiter des deutschen Informationsbüros des Europäischen Parlaments in Deutschland, Europäisches Parlament: Bürger-Handbuch, 6. Wahlperiode 2004 - 2009, Berlin 2005 (Stand: Februar 2005); Frank Piplat (Hrsg.), Leiter des deutschen Informationsbüros des Europäischen Parlaments in Deutschland, Europäisches Parlament: Bürger-Handbuch, 6. Wahlperiode 2009-2014, Berlin 2012 (Stand: April 2012). 
dem EP angehörten, bereits in der Hinsicht, dass diese Entscheidung nicht von der Unsicherheit einer weiteren Nominierung durch ihre jeweilige Partei oder den Ausgang der Wahlen zum EP abhing. Der Verzicht auf eine weitere Ausübung des Mandats im Laufe der Wahlperiode folgte häufig einer vorangehenden Entscheidung für ein anderes (politisches) Engagement. Es muss jedoch hier bereits einschränkend festgehalten werden, dass angesichts der insgesamt geringen Anzahl der während der Wahlperioden ausgeschiedenen Abgeordneten (insgesamt $46 \mathrm{MdEP}$ in sieben Wahlperioden, einschließlich der laufenden) substantielle Aussagen darüber, inwieweit und ob das EP-Mandat politische Karrieren begünstigte, kaum gewonnen werden können. ${ }^{17}$ Ebenso wenig ist es auf der Grundlage der auswertbaren Datenbasis möglich, einen intertemporalen Vergleich zwischen den Wahlperioden durchzuführen.

Tabelle 2 zeigt zunächst an, dass nur wenige Abgeordnete während der Wahlperiode ihr Mandat niederlegten, wenn außer den verstorbenen Abgeordneten noch einige „Sondereffekte“ herausgerechnet werden. Weitreichende Verallgemeinerungen lässt diese Datenbasis daher nur in der Richtung zu, dass es eine ausgeprägte Mandatsstabilität im EP gibt ${ }^{18}$, auch wenn das vorzeitige Ausscheiden mit jeweils über zehn Prozent der Abgeordneten in den ersten drei Wahlperioden hervorsticht. In der 1. Wahlperiode schieden neun Abgeordnete (11,1 Prozent) und in der 2. Wahlperiode zwölf Abgeordnete (14,8 Prozent) vorzeitig aus. Drei beziehungsweise fünf von ihnen verstarben. In der 1. Wahlperiode traten zwei Abgeordnete (die Gewerkschaftsfunktionäre Karl Hauenschild und Eugen Loderer) von ihrem Mandat noch im ersten Jahr der Wahlperiode zurück, da sie, nach eigenen Angaben, den mit dem Mandat verbundenen Arbeitsaufwand unterschätzt hätten. In der 2. Wahlperiode schieden drei Abgeordnete der Grünen vorzeitig aus, weil die Partei noch mit dem Konzept einer Ämterrotation experimentierte. Für die 1. und 2. Wahlperiode setzten vier MdEP ihre politische Karriere nach der Niederlegung des Mandats fort. Einer gab seinem bereits bestehenden Bundestagsmandat den Vorrang, drei traten von ihrem EP-Mandat zurück, weil sie während der Wahlperiode des EP in den Bundestag gewählt wurden. Dennoch sind ein Wechsel in ein anderes Parlament (Bundestag, Landesparlament) oder die Übernahme eines Regierungs- oder regierungsnahen Amtes für diese Gruppe der Abgeordneten marginale Ereignisse. ${ }^{19}$ Die Parlamentarier des Europäischen Parlaments scheinen nur in besonderen Situationen als Rekrutierungsreserve für exekutive Funktionen (Regierungsämter) genutzt

17 Vgl. aber die Hinweise von Richard Corbett / Francis Jacobs / Michael Shackleton, a.a.O. (Fn. 8), S. 54-61, die auf politische Erfahrungen in Parlamenten und Regierungen von EP-Abgeordneten ebenso hinweisen wie auf die Übernahme von hochrangigen Funktionen (Ministerposten) in nationalen Regierungen durch MdEP, nachdem ihr Mandat im EP endete, und dazu bemerken: „A stint as an MEP is a not infrequent part of a successful political career" (S. 60 f.).

18 Im Vergleich dazu errechnete Emil J. Kirchner, a.a.O. (Fn. 8), S. 6, S. 10 ff., bezogen auf alle MdEP für die ersten vier Jahre der ersten Wahlperiode eine insgesamt höhere Quote (24 Prozent) für alle ausgeschiedenen EP-Abgeordneten (und hochgerechnet für die gesamte Wahlperiode: 27,65 Prozent), bedingt vor allem durch den Wechsel in nationale Regierungen und Parlamente nach Wahlen und durch das von den Gaullisten angewandte Rotationsprinzip.

19 Vgl. hingegen die Ergebnisse ebenda, S. 10 f., der für alle vorzeitig ausgeschiedenen EP-Abgeordneten der 1. Wahlperiode (bis zum Stichtag 16. Juli 1983) einen Anteil von 37,89 Prozent ermittelt hat, die in eine nationale Regierung oder in ein nationales Parlament wechselten, wobei er insbesondere aufgrund der stattgefundenen nationalen Wahlen - eine hohe Konzentration bei den MdEP aus Belgien, Frankreich, Luxemburg und Irland feststellte. 
zu werden. Und selbst dies kann als eine Art „Sondereffekt“ einer historischen Ausnahmesituation angesehen werden. ${ }^{20}$

Die detaillierte Betrachtung der wenigen Daten und der „Sondereffekt“ bestätigt nochmals, dass für einzelne Wahlperioden verallgemeinerungsfähige quantitative Aussagen nicht gewonnen werden können. Möglich ist dies ansatzweise - wenngleich mit erheblichen Vorbehalten -, wenn die kumulierten Daten für die sieben Wahlperioden ausgewertet werden. $\mathrm{Zu}$ erkennen ist, dass der Tod immerhin bei 15 von insgesamt 46 ausgeschiedenen Abgeordneten (32,6 Prozent) die Mandatszeit beendete, während der Verzicht aus (vermutlich) Altersgründen und / oder Überlastung (6,5 Prozent) kaum ins Gewicht fiel. Die Übernahme von Regierungsfunktionen - überwiegend die Leitung von Landesministerien, während die Übernahme einer Funktion in einem Bundesministerium (Staatssekretär) nur einmal vorkam - spielte mit 17,4 Prozent (acht Fälle) eine ebenso große Rolle wie der Verzicht zugunsten eines Bundestagsmandats. Der Wechsel in ein Landesparlament (2,2 Prozent = 1) war indes marginal: Der „Aufstieg“ vom Landtag in das EP war und ist der üblichere Verlauf einer parlamentarischen Karriere und scheint mit einem Statusgewinn gegenüber einem Landtagsmandat verbunden zu sein. ${ }^{21}$

Insgesamt aber haben mindestens 21 (45,7 Prozent) Abgeordnete des Europäischen Parlaments, die ihr Mandat vor dem Ende der Wahlperiode niederlegten, ihre politische Karriere in einem Parlament, als Mitglied einer Regierung, in der Funktion eines Bürgermeisters / Oberbürgermeisters, in einer europäischen Institution (Generalanwalt am Europäischen Gericht) oder als Bevollmächtigter einer einigungsbedingten Bundeseinrichtung fortgesetzt. Für diese Gruppe der Abgeordneten war das EP nicht der Abschluss ihrer politischen Karriere, sondern (vielleicht) eine fördernde Zwischenstation. Darüber hinaus fanden sich zwei der wegen der Ämterrotation 1987 ausgeschiedenen Grünen zu Beginn der nächsten Wahlperiode wieder im Europäischen Parlament ein 22 , während der dritte „rotierte“ Grüne, Frank Schwalba-Hoth, eine erfolgreiche EG-/EU-orientierte Berater- und Lobbyisten-Karriere in Brüssel begann.

Wenn von den 46 im Laufe der sieben Wahlperioden ausgeschiedenen Abgeordneten die während der Mandatszeit Verstorbenen (15), die offensichtlich / vermutlich aus Altergründen ausgeschiedenen (zwei) sowie der eine Abgeordnete, der als „unklar“ rubriziert wurde, abgerechnet werden, lässt sich für alle übrigen freiwillig vorzeitig ausgeschiedenen MdEP (60,9 Prozent) feststellen, dass sie weiterhin professionell politisch (berufspolitisch) oder gesellschaftspolitisch aktiv waren. Es handelt sich aber um eine sehr kleine Gruppe. Von diesen Ergebnissen kann nicht auf eine markante personelle Zirkulation zwischen den Parlamenten im europäischen Mehrebenensystem geschlossen werden; ebenso wenig dienten die Abgeordneten des Europäischen Parlaments als wichtige Rekrutierungsreserve für Regierungsämter auf Bundes- oder Landesebene.

20 Der im Vergleich markante Wechsel von Abgeordneten in Regierungs- und regierungsnahe Funktionen während der 3. Wahlperiode (1989 bis 1994) resultierte vor allem aus der vereinigungsbedingten Nachfrage nach Politikern, von denen erwartet wurde, dass sie über die fachliche Expertise verfügten, um exekutive Aufgaben in den neuen Bundesländern übernehmen zu können.

21 Da Parlamentsmandate von engagierten Parteimitgliedern auf allen Ebenen nachgefragt und nicht selten in Konkurrenz vergeben werden, ist auch zu berücksichtigen, dass ein Wechsel in ein anderes Parlament in der Regel nur gegen erheblichen Widerstand der lokalen / regionalen Nominierungsgremien der Parteien durchsetzbar ist.

22 Dorothee Piermont und Friedrich-Wilhelm Graefe zu Baringdorf. 
Tabelle 2: Während der Wablperiode aus dem Europäischen Parlament ausgeschiedene Abgeordnete

\begin{tabular}{|c|c|c|c|c|c|c|c|c|c|}
\hline $\begin{array}{l}\text { Gründe des } \\
\text { Ausscheidens }\end{array}$ & $\begin{array}{c}\text { 1. WP } \\
(1979 \\
\text { seit } \\
1984)\end{array}$ & $\begin{array}{c}\text { 2. WP } \\
(1984 \\
\text { seit } \\
1989)\end{array}$ & $\begin{array}{c}\text { 3. WP } \\
(1989 \\
\text { seit } \\
1994)\end{array}$ & $\begin{array}{c}\text { 4. WP } \\
(1994 \\
\text { seit } \\
1999)\end{array}$ & $\begin{array}{c}\text { 5. WP } \\
(1999 \\
\text { seit } \\
2004)\end{array}$ & $\begin{array}{c}\text { 6. WP } \\
(2004 \\
\text { seit } \\
2009)\end{array}$ & $\begin{array}{l}\text { 7. WP } \\
(2009 \\
\text { bis } \\
2014)\end{array}$ & $\begin{array}{l}\text { ins- } \\
\text { gesamt }\end{array}$ & $\begin{array}{c}\text { in } \\
\text { Prozent }\end{array}$ \\
\hline $\begin{array}{l}\text { Wechsel in eine } \\
\text { Regierungsfunktion }\end{array}$ & & & $4 \mathrm{~g}$ & & $2^{\mathrm{n}}$ & $2^{\circ}$ & & 8 & 17,4 \\
\hline $\begin{array}{l}\text { Wechsel in den Bundes- } \\
\text { tag }\end{array}$ & $1^{\mathrm{a}}$ & $3^{d}$ & $1^{\mathrm{h}}$ & $1^{\mathrm{k}}$ & & $2 \mathrm{p}$ & & 8 & 17,4 \\
\hline $\begin{array}{l}\text { Wechsel in ein Landes- } \\
\text { parlament }\end{array}$ & & & & & & $1 \mathrm{q}$ & & 1 & 2,2 \\
\hline Ober-/Bürgermeister & & & $1^{\mathrm{i}}$ & $1^{1}$ & & & & 2 & 4,3 \\
\hline $\begin{array}{l}\text { Wechsel in eine } \\
\text { EU-Funktion }\end{array}$ & & & & $1 \mathrm{~m}$ & & & & 1 & 2,2 \\
\hline Sonstige & $3^{b}$ & $3 e$ & $1 j$ & & & & & 7 & 15,2 \\
\hline $\begin{array}{l}\text { Ausscheiden (vermutlich) } \\
\text { aus Altersgründen }\end{array}$ & $1^{\mathrm{c}}$ & $1^{f}$ & & & & & $1^{\mathrm{r}}$ & 3 & 6,5 \\
\hline $\begin{array}{l}\text { während Wahlperiode } \\
\text { verstorben }\end{array}$ & 3 & 5 & 3 & & 1 & 2 & 1 & 15 & 32,6 \\
\hline Unklar & 1 & & & & & & & 1 & 2,2 \\
\hline insgesamt & 9 & 12 & 10 & 3 & 3 & 7 & 2 & 46 & 100 \\
\hline MdEP insgesamt & 81 & 81 & 81 & 99 & 99 & 99 & 99 & & \\
\hline
\end{tabular}

Rundungsdifferenzen sind möglich.

a Zwar wechselte Herbert Köbler nicht in den Bundestag, sondern verzichtete auf sein EP-Mandat zugunsten seines seit 1972 (bis 1987) kontinuierlich gehaltenen Bundestagsmandates.

b Zwei Abgeordnete, Karl Hauenschild und Eugen Loderer, die führende Gewerkschaftsfunktionen bekleideten, traten von ihrem Mandat schon Anfang 1980 zurück; nach eigenen Angaben wegen der unerwartet hohen Arbeitsbelastung. Ein Abgeordneter, Erdmann Linde, gab sein Mandat 1981 ab, als er zum stellvertretenden Direktor der Volkshochschule Dortmund ernannt wurde.

c Vermutlich trat Willy Brandt (geboren 1913) 1983 aus Altersgründen und wegen Inanspruchnahme durch andere Aktivitäten von seinem EP-Mandat zurück; parlamentarisch gab er seinem Bundestagsmandat, das er bis zu seinem Tod 1992 besaß, den Vorrang.

d In den Bundestag wechselten: Fritz Gautier (SPD), Gero Pfennig (CDU), Heidemarie Wiecorek-Zeul (SPD).

e Drei Abgeordnete (Frank Schwalba-Hoth, Dorothea Piermont, Friedrich-Wilhelm Graefe zu Baringdorf) schieden während der Wahlperiode aus, weil ihre Partei Die Grünen in dieser Phase noch die Ämter-Rotation erprobte.

f Vermutlich aus Altersgründen legte Wilhelm F. T. Hahn (geboren 1909) Anfang Oktober 1987 sein EP-Mandat nieder.

g Vier MdEP wechselten in das Amt eines Ministers in einem Bundesland: Reinhold Bocklet, Werner Münch, Hartmut Perschau und Gerd Walter.

h Ein Abgeordneter, Günther Müller, war nur ersatzweise als Nachrücker im Europäischen Parlament, kontinuierlich hingegen Mitglied des Bundestages (1965 bis 1994).

i Eine Abgeordnete (Beate Weber) wurde zur Oberbürgermeisterin von Heidelberg gewählt.

j Ein Abgeordneter, Ludwig von Stauffenberg, wurde zum Bevollmächtigten einer Bundes-GmbH für die Privatisierung der Waldgebiete in den neuen Bundesländern ernannt.

k Claudia Roth (Die Grünen) übernahm ein Bundestagsmandat.

1 Heinke Salisch wurde zur Bürgermeisterin von Karlsruhe (Baudezernentin) gewählt.

m Siegbert Alber wechselte in die Funktion des Generalanwalts am Europäischen Gericht.

n Stanislav Tillich (CDU) wurde 1999 Staatsminister für Bundes- und Europaangelegenheiten in Sachsen; Emilia Müller (CSU) übernahm 2003 das Amt einer Staatssekretärin im Bayerischen Staatsministerium für Umwelt, Gesundheit und Verbraucher.

o Achim Laschet übernahm ein Landesministerium in NRW; Joachim Wuermelin wurde Staatssekretär im Bundesministerium für Wirtschaft und Technologie.

p Garrelt Duin und Ingo Schmitt traten als MdEP zurück, als sie Mitglieder des Bundestages wurden.

q Alexander Radwan wechselte als Abgeordneter in den Bayerischen Landtag.

r Kurt Lechner (CSU) (geboren 1942) legte sein Mandat vermutlich aus Altersgründen 2012 nieder.

Quelle: Vgl. Tabelle 1. 


\section{Ausscheiden aus dem Europäischen Parlament am Ende der Wahlperiode}

Da auch das Abgeordneten-Mandat im EP nicht grundsätzlich als Höhe- und Endpunkt einer Karriere als Berufspolitiker angesehen werden kann, liegt - wie eingangs betont - aus verschiedenen Gründen die Frage nach dem Verbleib der Parlamentarier nach dem Ausscheiden aus dem EP nahe. Da hier nur die Ergebnisse einer explorativ angelegten Untersuchung vorgestellt werden sollen, wurde aus forschungspragmatischen Gründen die Studie auf die Gruppe der Abgeordneten begrenzt, die mit dem Ende der 6. Wahlperiode (2009) aus dem EP ausgeschieden sind. ${ }^{23}$ Durch diese Einschränkung wurden insgesamt 41 Personen (41,4 Prozent der deutschen EP-Abgeordneten) erfasst, für die mit Hilfe einer Recherche über das Internet versucht wurde, die gewünschten Daten zum Verbleib zu ermitteln. ${ }^{24}$

Mit 41,4 Prozent aus dem Europäischen Parlament ausgeschiedenen Abgeordneten ${ }^{25}$ lag die Fluktuationsquote etwas höher (circa zehn Prozentpunkte) als im Durchschnitt der Wahlperioden, obwohl die EP-Wahl 2009 weder von einer Veränderung der im EP vertretenen Parteien noch von einer Veränderung der Anzahl der verfügbaren Parlamentssitze (1979: von 36 auf 81; 1994: von 81 auf 99) begleitet war (vgl. Tabelle 1). Ausschlaggebend für die höhere Fluktuation waren Mandatsverluste (bei der CDU) und ein auffällig umfangreicher Wechsel bei der Nominierung von MdEP-Kandidaten (vor allem PDS/Die Linke, Die Grünen und SPD) (vgl. Tabelle 3).

Das Ausscheiden aus dem Parlament kann auf unterschiedliche Weise und aus unterschiedlichen Gründen erfolgen. ${ }^{26}$ Grundsätzlich kann das Mandat freiwillig oder unfreiwillig beendet werden (vgl. Tabelle 4): Freiwillig erfolgt die Aufgabe des Mandats, wenn auf eigenen Wunsch auf eine erneute Nominierung verzichtet wird. Doch nicht immer ist eindeutig zu erkennen, ob der Verzicht tatsächlich ohne Einflussnahme oder Druck von Seiten

23 Durch die Beschränkung auf die 6. Wahlperiode müssen Abgeordnete nicht berücksichtigt werden, die Parteien angehörten, die in der nächsten Wahlperiode nicht mehr im Europäischen Parlament vertreten waren, da sich das Ensemble der im EP vertretenen Parteien von der 6. zur 7. Wahlperiode nicht verändert hatte.

24 Nicht für alle ausgeschiedenen Abgeordneten konnten die gewünschten Daten ermittelt werden, um die Fragen nach den Gründen für das Ausscheiden und nach den sich anschließenden (politischen) Aktivitäten beantworten zu können. Insgesamt betrifft dies acht Abgeordnete (19,5 Prozent). Von diesen acht Abgeordneten, die bei der folgenden Untersuchung weiterhin, soweit es möglich ist, einbezogen werden und unter denen bis auf die CSU allen anderen Parteien vertreten sind, waren vier zum Ende der Wahlperiode bereits 65 Jahre alt und älter. Die Annahme erscheint plausibel, dass deren Ausscheiden als Rückzug aus der aktiven Politik aufgefasst werden kann, weshalb mit Hilfe des Internets recherchierbare Informationen nicht ermittelt werden konnten. Auch für eine Abgeordnete der Grünen (Hiltrud Breyer, MdEP 1989 bis 2009) liegen keine eindeutigen Informationen für die Gründe ihres Ausscheidens vor; sie war für die EP-Wahl 2009 nominiert, erhielt mit Listenplatz 15 jedoch einen sehr unsicheren Listenplatz. Die Grünen konnten 2004 dreizehn Abgeordnete ins EP entsenden und erlangten 2009 vierzehn Sitze. Zu Hiltrud Breyer vgl. Benjamin Höhne, a.a.O. (Fn. 4), S. 305 f.

25 Faktisch kehrten 40 Abgeordnete nicht wieder in das EP zurück. Daniel Cohn-Bendit, der 2009 nicht mehr für die Grünen kandidierte, erlangte ein Mandat über die Liste von Les Verts / Europe Écologie in Frankreich. Er wird aber weiterhin mitgezählt, da er aus der Reihe der MdEP, die von deutschen Parteien nominiert wurden, ausschied.

26 Vgl. Michael Edinger / Bertram Schwarz, a.a.O. (Fn. 11), S. 13 - 19. 


\begin{tabular}{|c|c|c|c|c|c|c|}
\hline \multicolumn{7}{|c|}{$\begin{array}{l}\text { Tabelle 3: Am Ende der 6. Wahlperiode ausgeschiedene Abgeordnete } \\
\text { (2004 bis 2009, nach Parteien) }\end{array}$} \\
\hline & $\begin{array}{l}\text { ausgeschie- } \\
\text { dene } \\
\text { MdEP }\end{array}$ & $\begin{array}{l}\text { in Prozent } \\
\text { der MdEP } \\
\text { der jeweili- } \\
\text { gen Partei }\end{array}$ & $\begin{array}{c}\text { in Prozent } \\
\text { aller ausge- } \\
\text { schiedenen } \\
\text { MdEP } \\
(41=100 \\
\text { Prozent) }\end{array}$ & $\begin{array}{l}\text { Anteil an } \\
\text { allen MdEP } \\
(99=100 \\
\text { Prozent })\end{array}$ & $\begin{array}{l}\text { MdEP der } \\
\text { Parteien } \\
\text { 6. WP } \\
\text { (absolut) }\end{array}$ & $\begin{array}{l}\text { MdEP der } \\
\text { Parteien } \\
7 . \text { WP } \\
\text { (absolut) }\end{array}$ \\
\hline $\mathrm{CDU}$ & 14 & 35,0 & 34,1 & 14,1 & 40 & 34 \\
\hline CSU & 2 & 22,2 & 4,9 & 2,0 & 9 & 8 \\
\hline SPD & 10 & 43,5 & 24,4 & 10,1 & 23 & 23 \\
\hline Die Grünen & $8^{a}$ & 61,5 & 19,5 & 8,1 & 13 & 14 \\
\hline FDP & 1 & 14,3 & 2,4 & 1,0 & 7 & 12 \\
\hline PDS/Die Linke & 6 & 85,7 & 14,6 & 6,1 & 7 & 8 \\
\hline insgesamt & 41 & & 100 & 41,4 & 99 & 99 \\
\hline \multicolumn{7}{|c|}{$\begin{array}{l}\text { Rundungsdifferenzen sind möglich. } \\
\text { a Darunter auch Daniel Cohn-Bendit, der } 2009 \text { (7. Wahlperiode) für die französischen Les Verts / Euro- } \\
\text { pe Écologie in das Europäische Parlament einzog. } \\
\text { Quelle: Vgl. Tabelle 1. }\end{array}$} \\
\hline
\end{tabular}

der eigenen Fraktion oder Partei erfolgte. ${ }^{27}$ Ein freiwilliger Verzicht auf eine erneute Nominierung kann unterschiedliche Gründe haben, etwa das erreichte Alter oder eine angestrebte (politische) Funktion außerhalb des EP. Unfreiwilliges Ausscheiden aus dem EP nach dem Ende der Wahlperiode kann auf verschiedene Weise erfolgen:

- Die Fortsetzung des Mandats kann am (unerwarteten) Wählervotum scheitern (Stimmen- / Mandatsverluste der Partei), obwohl ein vermeintlich sicherer Listenplatz eingenommen wurde.

- Bei der parteiinternen Entscheidung über die Aufstellung der Kandidatenliste wurde ein ungünstiger und nicht aussichtsreicher Listenplatz zugewiesen.

- Eine erneute Nominierung wurde von den Parteigremien verweigert, die über die Kandidatenaufstellung und die Zusammensetzung der Liste entscheiden.

- Auf eine zwar gewünschte erneute Kandidatur wurde verzichtet, weil von der nominierenden Partei (aus politischen Gründen) nur ein abschätzbar erfolgloser Listenplatz angeboten wurde.

Tabelle 4 zeigt, dass etwas mehr als die Hälfte (53,7 Prozent) der ausgeschiedenen Abgeordneten in einer als freiwillig gewerteten Weise auf eine erneute Kandidatur für das EP verzichtet hat, ein erheblicher Teil vermutlich aus Altersgründen, auch wenn es für Parlamentarier keine normierte Altersbeschränkung gibt. ${ }^{28}$ Das unfreiwillige Ausscheiden resultierte

27 Vgl. Benjamin Höhne, a.a.O. (Fn. 4), S. 303, der am Beispiel von Ingo Friedrich, CSU, zum Zeitpunkt des Ausscheidens 67 Jahre und 30 Jahre MdEP, skizziert, dass Friedrichs Verzicht auf eine erneute Kandidatur eine verlorene Kampfabstimmung gegen einen konkurrierenden Bewerber vorausging, aber auch erforderlich war, um eine Gesamtliste der CSU abzusichern, bei der unterschiedliche regionale Interessen sowie die überraschende Nominierung von Monika Hohlmeier und ihre Platzierung auf dem sicheren Listenplatz 6 der CSU berücksichtigt werden mussten.

28 Es gibt eigentlich keinen überzeugenden Grund, für politische Mandatsträger eine mit Bezug auf die sozialversicherungsrechtliche Regelung bestimmte Altersgrenze, bis zu der politisches Engagement zu erwarten ist, festzulegen. Maria Kreiner, a.a.O. (Fn. 6); dies., a.a.O. (Fn. 11) schließt in 


\begin{tabular}{|c|c|c|c|c|c|c|c|c|}
\hline & $\mathrm{CDU}$ & CSU & SPD & $\begin{array}{c}\text { Die } \\
\text { Grünen }\end{array}$ & FDP & \begin{tabular}{|c|} 
PDS/ \\
Die Linke
\end{tabular} & $\begin{array}{c}\text { ins- } \\
\text { gesamt }\end{array}$ & $\begin{array}{c}\text { in } \\
\text { Prozent }\end{array}$ \\
\hline $\begin{array}{l}\text { freiwilliger Verzicht } \\
\text { auf Kandidatur }\end{array}$ & 12 & 1 & 4 & 3 & - & 2 & 22 & 53,7 \\
\hline $\begin{array}{l}\text { davon vermutlich } \\
\text { wegen Alters }\end{array}$ & 9 & $1 \mathrm{a}$ & 3 & - & - & - & 13 & 31,7 \\
\hline $\begin{array}{l}\text { unfreiwillig } \\
\text { davon: }\end{array}$ & 2 & 1 & 4 & 4 & - & 4 & 15 & 36,6 \\
\hline Wahlergebnis & 1 & 1 & - & - & & - & 2 & 4,9 \\
\hline $\begin{array}{l}\text { ungünstiger } \\
\text { Listenplatz (LP) }\end{array}$ & 1 & - & 3 & 2 & - & 1 & 7 & 17,1 \\
\hline $\begin{array}{l}\text { verweigerte / } \\
\text { gescheiterte } \\
\text { Nominierung }\end{array}$ & - & - & 1 & 2 & - & 3 & 7 & 17,1 \\
\hline $\begin{array}{l}\text { Verzicht auf } \\
\text { Nominierung w/ } \\
\text { ungünstigem LP }\end{array}$ & - & - & - & - & - & - & - & - \\
\hline sonstige / unklar & & & 2 & 1 & 1 & & 4 & 9,8 \\
\hline insgesamt & 14 & 2 & 10 & 8 & 1 & 6 & 41 & 100 \\
\hline \multicolumn{9}{|c|}{$\begin{array}{l}\text { Rundungsdifferenzen sind möglich. } \\
\text { a Die Einordnung von Ingo Friedrich (CSU) in diese Kategorie ist nicht zweifelsfrei, da er erst nach einer } \\
\text { Kampfabstimmung auf eine Nominierung verzichtete; er könnte auch in die Gruppe der „unfreiwilli- } \\
\text { gen“ unter „Verzicht auf Nominierung wegen ungünstigem LP“ eingeordnet werden, wenn diese Ka- } \\
\text { tegorie etwas erweitert würde (siehe Anmerkung 27). } \\
\text { Quelle: Vgl. Tabelle 1. }\end{array}$} \\
\hline
\end{tabular}

nur in wenigen Fällen (4,9 Prozent) aus dem Wählervotum. Ausschlaggebend oder zumindest vorentscheidend war der Beschluss der jeweiligen Parteigremien (34,2 Prozent) einen ungünstigen Listenplatzes zu vergeben, der entweder akzeptiert oder abgelehnt wurde, oder die Nominierung zu verweigern. Für einige Abgeordnete (9,8 Prozent) konnten entweder mit dem hier angewandten Recherche-Verfahren keine ausreichenden Informationen gewonnen werden (unklar) oder die Gründe für den Verzicht auf eine erneute Kandidatur changierten zwischen persönlichen Motiven (Rückzug), im Vorfeld bereits verweigerter erneuter Nominierung durch die Partei oder anderen politischen Optionen (sonstige). ${ }^{29}$

ihrer Studie Personen, die das sozialversicherungsmäßige Renteneintrittsalter erreicht haben, aus ihrer Untersuchung aus. Diese Entscheidung ist bestenfalls dann zu rechtfertigen, wenn die Problematisierung der Versorgungssituation ein zentrales Anliegen einer Untersuchung zum „Amt auf Zeit" ist. Wenn indes vom politischen Charakter des Parlamentsmandates ausgegangen wird, gibt es eine altersbezogene Limitierung nur für den „Eintritt“, für den Beginn des passiven Wahlrechts, aber keine altersbezogene Begrenzung für die Ausübung des Abgeordneten-Mandats, für die Beendigung des Mandats und allgemein für politisches Engagement. Die hier vorgestellten Ergebnisse zum „Verbleib“ der Europa-Abgeordneten lassen erkennen, dass mit einer solchen altersbezogenen Begrenzung zahlreiche politisch relevante Folgeaktivitäten von Abgeordneten unberücksichtigt blieben.

29 Als unklar wird das Ausscheiden von Helmut Kuhne und Ralf Walter (beide SPD) angesehen; beide waren noch nicht 60 Jahre zum Zeitpunkt des Ausscheidens aus dem EP und nicht erneut nominiert worden. Unter „sonstige“ werden Willem Schuth (FDP) und Milan Horáček (Die Grü- 


\subsection{Freiwilliger Verzicht auf das Mandat / Alter als Grund für Nicht-Nominierung}

Eine Gruppe von Abgeordneten (neun MdEP), die freiwillig auf ihr Mandat verzichteten, verfolgte erkennbar und überwiegend eindeutig berufspolitischen Perspektiven, indem das politische Engagement in anderen Parlamentsmandaten, in einer hohen und einflussreichen Parteifunktion, in Regierungsämtern (sechs) oder in gesellschaftspolitisch relevanten ehrenamtlichen Positionen (drei) fortgesetzt wurde. ${ }^{30}$

Die Mehrzahl der ausgeschiedenen Abgeordneten (31) wurde - aus unterschiedlichen Gründen - nicht mehr nominiert. Das eigene Alter war sicherlich bei einigen ein Grund, auf eine erneute Kandidatur zu verzichten oder von Seiten der entscheidenden Parteigremien eine erneute Nominierung zu verweigern. Es war aber keineswegs der einzige oder insgesamt vorrangige Grund. Bereits die Altersstruktur aller ausgeschiedenen Abgeordneten (vgl. Tabelle 5) verdeutlicht, dass nur etwa 50 Prozent der ausgeschiedenen zum Ende der Wahlperiode 60 Jahre und älter waren (21 Abgeordnete, davon acht jünger als 65 Jahre) und nur fünf Prozent (also zwei) das 70. Lebensjahr erreicht oder überschritten hatten.

Alter als Anlass, die Karriere als Berufspolitiker zu beenden, scheint nur für wenige Abgeordnete der ausschlaggebende Grund gewesen zu sein, auf das Mandat und auf eine weitere Nominierung zu verzichten.

Die Gruppe der Abgeordneten, die freiwillig aus dem EP ausschieden und von denen angenommen werden kann, dass sie vor allem aus Altersgründen ihre berufspolitische / parlamentarische Aktivität beendeten, umfasst (mindestens) dreizehn Personen. ${ }^{31}$ Von diesen waren zehn zum Zeitpunkt des Ausscheidens 65 Jahre und älter und drei hatten das 60 . Lebensjahr bereits überschritten. Aber nur ein Teil dieser dreizehn ausgeschiedenen Abgeordneten zog sich tatsächlich aus der Politik zurück. ${ }^{32}$ Für sechs Parlamentarier konnten hingegen die Übernahme ehrenamtlicher Parteifunktionen, Engagement in lobbyistischen Vereinigungen, Beratungstätigkeit mit europapolitischem Bezug oder unternehmerische Aktivitäten festgestellt werden. Und zumindest für einen dieser Abgeordneten (Ingo Friedrich, CSU) kann angenommen werden, dass der Verzicht auf eine Kandidatur nicht völlig freiwillig erfolgte. 33

Markant ist bei der Gruppe der Abgeordneten, die freiwillig auf eine Fortsetzung des Mandats verzichteten, die zeitliche Intensität der parlamentarischen Erfahrungen. Von diesen 22 MdEP waren vier nur fünf Jahre im EP gewesen, und eine Abgeordnete gehörte dem

nen) gefasst. Schuth trat angeblich aus „familiären Gründen“ nicht wieder an; da er unzulänglich in der Partei vernetzt war, konnte Gesine Meißner, die mit ihm um die Nominierung konkurrierte, bereits im Vorfeld der Kandidatenaufstellung innerparteilich den Vorrang gewinnen, vgl. Benjamin Höhne, a.a.O. (Fn. 4), S. 304. Horáček war für eine grüne Partei in Tschechien auf einem wenig aussichtsreichen Listenplatz nominiert worden, nachdem anscheinend bereits in der „Vorentscheidungsarena" von den Grünen auf einen Verzicht auf eine erneute Kandidatur gedrängt worden war, ebd., S. 305.

30 Nicht immer ist eindeutig zu entscheiden, ob beispielsweise der Vorsitz im Rundfunkrat des WDR anstelle des Abgeordnetenmandats angestrebt oder als Kompensation nach einem partei-gewünschten Mandatsverzicht zugeteilt wurde.

31 Diese Altersgruppe würde durch Abgeordnete, die in der Kategorie „unklar“ untergebracht wurden, nicht verändert.

32 Für sieben Abgeordnete, von denen der Rückzug aus der Politik angenommen wird, konnten keine aktuellen politischen Aktivitäten recherchiert werden.

33 Vgl. Michael Edinger / Bertram Schwarz, a.a.O. (Fn. 11), S. 13 - 19. 


\begin{tabular}{|c|c|c|c|c|c|c|c|}
\hline \multicolumn{8}{|c|}{$\begin{aligned} \text { Tabelle 5: Altersstruktur der mit dem Ende der 6. Wahlperiode } 2009 \text { ausgeschiedenen } \\
\text { Abgeordneten (in Prozent, absolut nach Parteien) }\end{aligned}$} \\
\hline & $\begin{array}{c}\text { ausge- } \\
\text { schiedene } \\
\text { MdEP }\end{array}$ & $\begin{array}{c}\text { in Prozent } \\
(41=100 \\
\text { Prozent })\end{array}$ & $\begin{array}{l}\text { CDU } / \\
\text { CSU }\end{array}$ & SPD & $\begin{array}{c}\text { Die } \\
\text { Grünen }\end{array}$ & FDP & $\begin{array}{c}\text { PDS/ } \\
\text { Die Linke }\end{array}$ \\
\hline 30 bis 39 Jahre & 1 & 2,4 & - & - & - & - & 1 \\
\hline 40 bis 49 Jahre & 3 & 7,3 & - & - & 1 & - & 2 \\
\hline 50 bis 59 Jahre & 16 & 39,0 & 3 & 6 & 3 & 1 & 3 \\
\hline 60 bis 64 Jahre & 8 & 19,5 & 5 & 1 & 2 & - & - \\
\hline 65 bis 69 Jahre & 11 & 26,8 & 7 & 2 & 2 & & \\
\hline 70 bis 79 Jahre & 2 & 4,9 & 1 & 1 & - & - & - \\
\hline insgsamt & 41 & 100 & 16 & 10 & 8 & 1 & 6 \\
\hline
\end{tabular}

EP vor dem Ausscheiden „lediglich“ zehn Jahre an. Die übrigen 17 waren mehr als zehn und bis zu 35 Jahre Mitglied eines Parlaments. Allein dreizehn Parlamentarier gehörten nur dem EP drei und mehr Wahlperioden an. Das Ausscheiden der meisten Abgeordneten beendete daher eine doch sehr lange berufspolitische Parlamentstätigkeit, die von den meisten überwiegend im EP verbracht worden war.

Die freiwillig ausscheidenden Abgeordneten, die nur eine Wahlperiode Mitglieder des EP waren, waren entweder keine Berufspolitiker (Vural Öger, Kurt Joachim Lauk) oder hatten ihren politisch-parlamentarischen Handlungsschwerpunkt in der Bundespolitik. Das EPMandat war für sie von vornherein eine zeitlich begrenzte politische Zwischenstation gewesen (Sarah Wagenknecht, Frithjof Schmidt, Cem Özdemir). 34

\subsection{Erneute Kandidatur}

Von den 41 ausgeschiedenen Abgeordneten waren zehn MdEP35 noch für die Wahl 2009 nominiert (vgl. Tabelle 6). Zum Zeitpunkt des Ausscheidens waren diese zwischen 44 und 65 Jahre alt (sechs waren in den 50er Jahren und drei in den 60er Jahren). Einige der Kandidaten verpassten die Wiederwahl sehr knapp; zumindest zwei hatten mit einer Erneuerung ihres Mandats rechnen können. ${ }^{36}$ Bei anderen Abgeordneten mag der zugewiesene Listenplatz ein (vielleicht sanfter) Weg des Mandatsentzugs gewesen sein. ${ }^{37}$ Eine explizit politisch

34 Im Grunde könnte auch Horst Posdorf (CDU), der 2005 als Nachrücker in das EP einzog und vorher 15 Jahre Mitglied des Landtags von Nordrhein-Westfalen war, zu dieser Gruppe gezählt werden.

35 Einschließlich Daniel Cohn-Bendit (siehe Fußnote 25).

36 Dazu gehören Christoph Konrad (CDU) und Gabriele Stauner (CSU), während Roland Gewalt, der 2005 als Nachrücker in das EP kam und auf dem zweiten Platz der Berlin CDU-Liste stand, nicht mehr mit einer Wahl ins EP rechnen konnte. Bei der Zuordnung der Listenplätze für diese Kandidaten spielte jeweils der innerparteiliche regionale Proporz eine wichtige Rolle; vgl. Benjamin Höhne, a.a.O. (Fn. 4), S. $270-273$, S. 302 f.

37 Hohe Erwartung auf einen Wiedereinzug ins EP konnten sich die SPD-Abgeordneten mit den Listenplätzen 24 bis 26 (Erika Mann, Karin Jöns und Ulrich Stockmann) bei der Wahl 2009 kaum 


\begin{tabular}{|c|c|c|c|c|}
\hline \multicolumn{5}{|c|}{$\begin{array}{l}\text { Tabelle 6: Für die Wahl zum Europäischen Parlament } 2009 \text { nominierte, aber ausgeschiedene } \\
\text { Abgeordnete (nach Parteien) }\end{array}$} \\
\hline & $\begin{array}{c}\text { Abgeordnete: } \\
\text { nominiert und } \\
\text { ausgeschieden }\end{array}$ & $\begin{array}{c}\text { ausgeschiedene } \\
\text { Abgeordnete } \\
\text { insgesamt }\end{array}$ & $\begin{array}{l}\text { Sitze im EP } \\
\quad 2004\end{array}$ & $\begin{array}{l}\text { Sitze im EP } \\
\quad 2009\end{array}$ \\
\hline $\mathrm{CDU}$ & 2 & 14 & 40 & 34 \\
\hline CSU & 1 & 2 & 9 & 8 \\
\hline SPD & 3 & 10 & 23 & 23 \\
\hline Die Grünen & $2^{a}$ & 8 & 13 & 14 \\
\hline FDP & - & 1 & 7 & 12 \\
\hline PDS/Die Linke & 1 & 6 & 7 & 8 \\
\hline insgesamt & 9 & 41 & 99 & 99 \\
\hline
\end{tabular}

motivierte Entscheidung für die Zuweisung eines aussichtslosen Listenplatzes ist bei einem PDS-Kandidaten (Tobias Pflüge) zu vermuten ${ }^{38}$, vielleicht auch (aber aus anderen Gründen) bei der ungünstigen Platzierung einer Abgeordneten der Grünen (Hiltrud Breyer). Das Alter der Kandidaten spielte nur bei den Grünen insofern eine Rolle, als bei der Listenaufstellung auch eine Art Generationengerechtigkeit berücksichtigt werden sollte und damit eine „Verjüngung" der Kandidaten und Abgeordneten erfolgte.

Die Mehrzahl dieser ausgeschiedenen nominierten Parlamentarier blieb weiterhin in Parteigremien politisch aktiv. Drei waren darüber hinaus noch als alimentierte Berufspolitiker engagiert: Eine ausgeschiedene Abgeordnete erlangte ein Landtagsmandat ${ }^{39}$, eine andere wechselte in die administrative Position der Amtschefin einer Landesministerin in Bayern ${ }^{40}$, ein weiterer wurde zum Landesbeauftragten für die Stasi-Unterlagen gewählt. ${ }^{41}$ Nicht überraschen sollte es, dass die im EP gewonnenen Kompetenzen und Verbindungen für eine Intensivierung von (bereits ausgeübten) lobbyistischen Aktivitäten in Brüssel genutzt wur-

machen, denen im Vergleich zur Kandidaten-Liste von 2004 ungünstigere Plätze zugewiesen wurden, zum Teil als Folge eines neuen regionalen Verteilungsschlüssels bei der Aufstellung der SPDBundesliste für die Europawahl; vgl. Benjamin Höhne, a.a.O. (Fn. 4), S. 273 ff., S. 303 f. Auch Hiltrud Breyer und Gisela Kallenbach von den Grünen konnten mit ihren Listenplätzen nicht mit einem erneuten Einzug in das EP rechnen; zu den möglichen Motiven für die ungünstige Platzierung vgl. ebenda, S. 285 f. Mit Ausnahme von Gisela Kallenbach, die nur in der 6. Wahlperiode ein Mandat im EP hatte, gehörten die genannten Abgeordneten 15 Jahre (Mann, Jöns, Stockmann) oder sogar 20 Jahre (Breyer) dem EP an. Zur Bewertung der Wahlaussichten und des Wahlverlaufs vgl. Oskar Niedermayer, a.a.O. (Fn. 2), S. $711-731$.

38 Seine Nominierung auf dem 10. Listenplatz deutet darauf hin. Die PDS tauschte für die Wahl 2009 fast ihr gesamtes EP-Personal aus (von sieben MdEP wurden fünf nicht mehr nominiert, und nur eine Abgeordnete, Gabi Zimmer, schaffte den Wiedereinzug ins EP, obwohl die PDS 2009 einen Sitz hinzugewinnen konnte) vor allem wegen Differenzen mit den europapolitischen Positionen und Vorgaben der PDS und als Folge der innerparteilichen Fragmentierung; vgl. Benjamin Höhne, a.a.O. (Fn. 4), S. 302, S. 306 ff.

39 Gisela Kallenbach, Die Grünen, in Sachsen 2009.

40 Gabriele Stauner, CSU, seit März 2011.

41 Ulrich Stockmann, SPD, in Sachsen-Anhalt; er konnte diese Position jedoch bislang wegen Konkurrenzklagen nicht antreten. 
den. ${ }^{42}$ Auch das - vielleicht nicht immer erwartete - Ausscheiden aus dem EP führte, soweit erkennbar, für die Mehrzahl dieser neun (beziehungsweise zehn) Abgeordneten zu weiterem politischen, administrativen oder lobbyistischen Handeln. Nur von einem nominierten und ausgeschiedenen Abgeordneten ist bekannt, dass er sich aus der aktiven Politik zurückgezogen hat. ${ }^{43}$

\subsection{Verweigerte / gescheiterte Nominierung und Verzicht auf eine Kandidatur}

Für wenige Abgeordnete ist festzustellen, dass sie weiterhin für eine Kandidatur bereitstanden, aber aus unterschiedlichen Gründen von den zuständigen Parteigremien nicht wieder nominiert wurden. Dafür müssen nicht immer politische Differenzen ausschlaggebend gewesen sein. ${ }^{44}$ Eine als sehr lang empfundene Parlamentstätigkeit, deren Fortsetzung eine gewünschte personelle Erneuerung blockierte, konnte als Grund ausreichen, um eine Wiederaufstellung zu verhindern. ${ }^{45}$

Als negative politische Stellungnahme der Parteimehrheit beziehungsweise der Mehrheit des nominierenden Parteigremiums musste hingegen eine gescheiterte Bewerbung für einen aussichtsreichen Listenplatz verstanden werden (eine Variante wäre das Angebot eines aussichtslosen Listenplatzes), wenn mit diesem Nominierungskonflikt eine offen ausgetragene Kritik an den europapolitischen Positionen, die während der Wahlperiode im EP vertreten wurden und die von der Beschlusslage der Partei abwichen, verbunden war. ${ }^{46}$

42 Erika Mann, SPD, fungiert inzwischen als Leiterin des Brüsseler Büros des Unternehmens Facebook.

43 Roland Gewalt schied mit 51 Jahren aus der aktiven Politik aus; er war zu diesem Zeitpunkt bereits 19 Jahre berufspolitisch als Parlamentarier im Berliner Abgeordnetenhaus, im Bundestag und schließlich im Europäischen Parlament engagiert gewesen.

44 Dies dürfte jedoch bei Angelika Beer (53, Die Grünen) der Fall gewesen sein, die zum Zeitpunkt des Ausscheidens aus dem EP auf insgesamt bereits 16 Jahre Parlamentstätigkeit, fünf davon im Europäischen Parlament, zurückschauen konnte. Die verweigerte Nominierung motivierte ihren späteren Wechsel zur Piratenpartei und die erfolgreiche Kandidatur für den Landtag von Schleswig-Holstein 2012. Vgl. Benjamin Höhne, a.a.O. (Fn. 4), S. 305.

45 Das scheint für Lieselotte (Lissy) Gröner (55, SPD) zuzutreffen, die 20 Jahre, von 1989 bis 2009, MdEP war. Mit Variationen gilt dies auch für Friedrich-Wilhelm Graefe zu Baringdorf (66, Die Grünen), der auf insgesamt 23 Abgeordnetenjahre im Europäischen Parlament zurückblicken konnte. Bei der SPD führte eine Stagnation bei der personellen Innovation in den beiden zurückliegenden Wahlperioden (1999: vier neue Abgeordnete, 2004: nur einer), bei gleichzeitigem Rückgang der Anzahl der Sitze von 1999: 33 auf 2004/2009: 23 zu einem personellen Erneuerungsbedarf. Er wurde 2009 in der Weise umgesetzt, dass zehn Abgeordnete ausschieden und ersetzt wurden.

46 Für eine erneute Nominierung für einen Listenplatz hatten sich die beiden PDS-Abgeordneten Sylvia-Yvonne Kaufmann (54) und André Brie (59) beworben; sie waren in den Abstimmungen der Parteigremien jedoch gescheitert, obwohl sie für die Wahl 2004 noch Spitzenpositionen auf der Kandidatenliste eingenommen hatten. Brie setzte seine politische Karriere in der PDS/Die Linke später als MdL in Mecklenburg-Vorpommern fort, Kaufmann wechselte zur SPD und ist bemüht, für die kommende Wahl zum Europäischen Parlament im Jahr 2014 nominiert zu werden; vgl. auch Benjamin Höhne, a.a.O. (Fn. 4), S. 306 f. 


\section{Nach dem Mandat}

Die unterschiedlichen Gründe, weshalb auf eine erneute Kandidatur verzichtet wurde oder eine Erneuerung des Parlamentsmandats misslang, weisen bereits darauf hin, dass mit dem Ausscheiden aus dem Europäischen Parlament nicht gleichzeitig das politische und gesellschaftspolitische Engagement beendet ist oder eine Karriere als Berufspolitiker abgeschlossen wurde. Bereits die Altersstruktur der ausgeschiedenen MdEP lässt für viele eine weitere (berufs-)politische Tätigkeit erwarten und für andere (ältere) zumindest eine ehrenamtliche Fortsetzung des bisherigen politischen Engagements vermuten. Die lange Verweildauer vieler ausgeschiedener Abgeordneten in Parlamenten weist auch darauf hin, dass sich die meisten Abgeordneten als Berufspolitiker verstanden und politisches Engagement ihren Lebensmittelpunkt bildete (zur Professionalisierung der Abgeordneten vgl. Tabelle 8 und 9). Zusammenfassend sollen daher die oben nur beiläufig erwähnten politischen und gesellschaftspolitischen Aktivitäten der Abgeordneten nach dem Ende des EP-Mandats dargestellt werden.

Nicht für alle ausgeschiedenen Abgeordneten ließen sich politische oder gesellschaftspolitische Aktivitäten recherchieren (vgl. zum folgenden Tabelle 7). Für sechs Abgeordnete (14,6 Prozent) kann angenommen werden, dass sie sich mit dem Ausscheiden aus dem EP aus Altersgründen auch aus der (aktiven) Politik weitgehend zurückgezogen haben. ${ }^{47}$ Für eine weitere Gruppe von acht Abgeordneten (19,5 Prozent) konnten keine weiteren politischen / gesellschaftspolitischen Aktivitäten recherchiert werden, obwohl von ihnen aufgrund ihres Alters weiterhin irgendeine Art von Engagement zu erwarten gewesen wäre. Weitere zwei Abgeordnete (4,9 Prozent) haben Aufgaben in Unternehmen übernommen oder fortgesetzt.

Bemerkenswert - aber angesichts einer langen politischen Karriere nicht überraschend - ist, dass elf Abgeordnete (26,8 Prozent) weiterhin im politischen oder politisch-administrativen Bereich einflussreiche Funktionen übernahmen (Parlamentsmandate, Regierungsfunktionen, hohe Parteifunktionen). Da nicht anzunehmen ist, dass solche Positionen vorrangig zur „Versorgung“ von ausgeschiedenen Abgeordneten bereitgestellt wurden ${ }^{48}$, dürften sich die Abgeordneten nicht zuletzt durch ihre europapolitischen Kompetenzen für die neuen Funktionen qualifiziert haben. ${ }^{49}$ Darüber hinaus findet sich eine Anzahl von ehemaligen EP-Mitgliedern, obwohl diese überwiegend kein weiteres Mandat mehr anstrebten (zum Teil aus Altersgründen und nach einer langen parlamentarischen Karriere) ${ }^{50}$, in ehren-

47 Der Rückzug aus der aktiven Politik muss nicht notwendig auch den Verzicht auf zum Beispiel ehrenamtliches kommunalpolitisches Engagement einschließen. Mit der angewandten einfachen Internetrecherche sind solche Aktivitäten jedoch nur zu erfassen, wenn eine aktuelle persönliche Homepage unterhalten wird.

48 Zur geringen Unterstützung, die Abgeordnete nach ihrem Ausscheiden von ihren Parteien erhielten, vgl. Maria Kreiner, a.a.O. (Fn. 6), S. 78 - 85; Michael Edinger / Bertram Schwarz, a.a.O. (Fn. 11), S. $37 \mathrm{f}$.

49 Für einige Abgeordnete (siehe oben, bei Fußnote 34) kann angenommen werden, dass die Mandatszeit im Europäischen Parlament als Interim verstanden wurde, bis eine eigentlich angestrebte und als politisch einflussreicher angesehene Position zu erreichen war.

50 Zwei von den sechs Abgeordneten dieser Gruppe, Sylvia-Yvonne Kaufmann (PDS/SPD) und Tobias Pflüger (PDS) streb(t)en allerdings neben ehrenamtlichen Parteifunktionen weiterhin ein politisches Mandat an. Kaufmann im Europäischen Parlament, Pfiüger im Bundestag. 


\begin{tabular}{|c|c|c|c|c|c|c|c|}
\hline & $\begin{array}{c}\text { ins- } \\
\text { gesamt }\end{array}$ & $\begin{array}{l}\text { in Prozent } \\
(41=100)\end{array}$ & $\begin{array}{l}\mathrm{CDU} / \\
\mathrm{CSU}\end{array}$ & SPD & $\begin{array}{c}\text { Die } \\
\text { Grünen }\end{array}$ & FDP & \begin{tabular}{|c|} 
PDS/ \\
Die Linke
\end{tabular} \\
\hline $\begin{array}{l}\text { Parlamentsmandat (MdB } \\
\text { / MdL / MdEP) }\end{array}$ & 7 & 17,1 & 1 & & $4^{a}$ & - & 2 \\
\hline $\begin{array}{l}\text { Regierungsfunktion / } \\
\text { regierungsnahe Funk- } \\
\text { tion }^{\text {b }}\end{array}$ & 3 & 7,3 & 1 & $1 \mathrm{c}$ & - & - & 1 \\
\hline $\begin{array}{l}\text { hohe Parteifunktion } \\
\text { (hauptamtlich) }\end{array}$ & 1 & 2,4 & - & - & 1 & - & - \\
\hline $\begin{array}{l}\text { Parteifunktionen } \\
\text { (ehrenamtlich) }\end{array}$ & 6 & 14,6 & 3 & 1 & - & - & $2^{e}$ \\
\hline $\begin{array}{l}\text { gesellschaftspolitische } \\
\text { Funktionend }\end{array}$ & 2 & 4,9 & 1 & 1 & & & \\
\hline $\begin{array}{l}\text { europapolitisches } \\
\text { Engagement }\end{array}$ & 3 & 7,3 & 2 & 1 & - & - & - \\
\hline $\begin{array}{l}\text { lobbyistische Funktio- } \\
\text { nen / Tätigkeit }\end{array}$ & 3 & 7,3 & 1 & 1 & 1 & - & - \\
\hline $\begin{array}{l}\text { aus der Politik ausge- } \\
\text { schieden (vor allem } \\
\text { wegen Alter) }\end{array}$ & 6 & 14,6 & 5 & 1 & - & - & - \\
\hline Sonstiges & 2 & 4,9 & 1 & 1 & - & & \\
\hline Unklar & 8 & 19,5 & 1 & 3 & 2 & 1 & 1 \\
\hline insgesamt & 41 & 100 & 16 & 10 & 8 & 1 & 6 \\
\hline \multicolumn{8}{|c|}{$\begin{array}{l}\text { Rundungsdifferenzen sind möglich. } \\
\text { a Davon Daniel Cohn-Bendit } 2009 \text { für Les Verts / Europe Écologie (Frankreich); Angelika Beer } 2012 \text { für } \\
\text { die Piraten MdL in Schleswig-Holstein. } \\
\text { b Außer Landesminister werden hier auch hohe Funktionen in einem Ministerium (Amtschefin) und } \\
\text { Leitungen von Bundes- oder Landesbehörden (Landesbeauftragter für Stasi-Unterlagen) zusammenge- } \\
\text { fasst. } \\
\text { c Ein ausgeschiedener Abgeordneter (Ulrich Stockmann) wurde } 2010 \text { als Landesbeauftragter für die } \\
\text { Stasi-Unterlagen (Brandenburg) vom Landtag gewählt, die Amtsübernahme scheiterte jedoch aufgrund } \\
\text { von Konkurrenten-Klagen. } \\
\text { d Darunter auch hochrangige Funktionen wie der Vorsitz des WDR-Rundfunkrates. } \\
\text { e Eine PDS-Abgeordnete, Sylvia-Yvonne Kaufmann, wechselte, nachdem sie nicht auf einen aussichtsrei- } \\
\text { chen Listenplatz nominiert wurde, noch im Jahr } 2009 \text { zur SPD; sie bewirbt sich - derzeit - für eine } \\
\text { Nominierung auf der SPD-Liste für die Wahl zum Europäischen Parlament } 2014 \text {. } \\
\text { Quelle: Vgl. Tabelle 1. }\end{array}$} \\
\hline
\end{tabular}

amtlichen Funktionen in ihrer Partei (14,6 Prozent), in Wohlfahrtsorganisationen oder in gehobener Beiratsposition (4,9 Prozent) wieder, oder sie nutzen ihre Erfahrungen aus dem EP für ein ehrenamtliches oder beratendes europapolitisches Engagement (7,3 Prozent). Eine Ausnahme stellt hingegen die Nutzung der erworbenen europapolitischen Kompetenzen für einen Wechsel zu einer unternehmens- oder verbände-lobbyistischen Tätigkeit dar (7,3 Prozent).

Insgesamt zeigt die Übersicht über den Verbleib der aus dem Europäischen Parlament ausgeschiedenen Abgeordneten, dass ein ausgeprägtes Bestreben bestand, das bisherige politisch-parlamentarische Engagement in anderer Weise fortzusetzen. Eine „natürliche“ Altersgrenze gab es dabei nicht. Diese findet sich - wie bereits angemerkt - eher in einer 


\begin{tabular}{|c|c|c|c|c|c|c|c|c|}
\hline & $\begin{array}{l}\text { bis } \\
\text { Jahre }\end{array}$ & $\begin{array}{c}\text { MdEP } \\
\text { insgesamt }\end{array}$ & $\begin{array}{c}\text { in } \\
\text { Prozent }\end{array}$ & $\begin{array}{c}\mathrm{CDU} / \\
\mathrm{CSU}\end{array}$ & SPD & $\begin{array}{c}\text { Die } \\
\text { Grünen }\end{array}$ & FDP & $\begin{array}{c}\text { PDS/ } \\
\text { Die Linke }\end{array}$ \\
\hline $\begin{array}{l}\text { 1. bis 6.WP } \\
\text { (1979 bis 2009) }\end{array}$ & 30 & 3 & 7,3 & 2 & 1 & - & - & - \\
\hline $\begin{array}{l}\text { 2. bis 6. WP } \\
\text { (1984 bis 2009) }\end{array}$ & 25 & $2^{a}$ & 4,9 & - & 1 & 1 & - & - \\
\hline $\begin{array}{l}\text { 3. bis 6. WP } \\
\text { (1989 bis 2009) }\end{array}$ & 20 & $4^{b}$ & 9,8 & 2 & 1 & 1 & - & - \\
\hline $\begin{array}{l}\text { 4. bis 6. WP } \\
\text { (1994 bis 2009) }\end{array}$ & 15 & $13^{c}$ & 31,7 & 6 & 6 & 1 & - & - \\
\hline $\begin{array}{l}\text { 5. bis } 6 \text { WP } \\
\text { (1999 bis 2009) }\end{array}$ & 10 & 7 & 17,1 & 3 & - & - & - & 4 \\
\hline $\begin{array}{l}\text { 6. WP } \\
\text { (2004 bis 2009) }\end{array}$ & 5 & $12^{d}$ & 29,3 & 3 & 1 & 5 & 1 & 2 \\
\hline MdEP insgesamt & & 41 & 100 & 16 & 10 & 8 & 1 & 6 \\
\hline \multicolumn{9}{|c|}{$\begin{array}{l}\text { Rundungsdifferenzen sind möglich. } \\
\text { a Davon ein MdEP vorzeitig in 2. Wahlperiode ausgeschieden, in EP: } 1984 \text { bis } 1987 \text { und } 1989 \text { bis } 2009 . \\
\text { b Davon ein MdEP als Nachrücker in EP: } 1991 \text { bis } 2009 . \\
\text { c Davon ein MdEP auch in 7. Wahlperiode im EP. } \\
\text { d Davon zwei MdEP als Nachrücker in EP: } 2005 \text { bis } 2009 . \\
\text { Quelle: Vgl. Tabelle 1. }\end{array}$} \\
\hline
\end{tabular}

Tabelle 9: Parlamentserfahrung (insgesamt) der 2009 aus dem EP ausgeschiedenen Abgeordneten (nach Parteien und Jahren)

\begin{tabular}{|l|c|c|c|c|c|c|c|}
\hline $\begin{array}{l}\text { Mitgliedszeit in } \\
\text { Parlamenten }\end{array}$ & $\begin{array}{c}\text { Anzahl } \\
\text { der MdEP }\end{array}$ & $\begin{array}{c}\text { in } \\
\text { Prozent }\end{array}$ & $\begin{array}{c}\text { CDU/ } \\
\text { CSU }\end{array}$ & SPD & $\begin{array}{c}\text { Die } \\
\text { Grünen }\end{array}$ & FDP & $\begin{array}{c}\text { PDS/ } \\
\text { Die Linke }\end{array}$ \\
\hline 1 bis 5 Jahre & 7 & 17,1 & 1 & 1 & 2 & 1 & 2 \\
6 bis 10 Jahre & 6 & 14,6 & 2 & - & 1 & - & 3 \\
11 bis 15 Jahre & $10^{a}$ & 24,4 & 4 & 4 & $2^{\text {a }}$ & - & - \\
16 bis 20 Jahre & 10 & 24,4 & 4 & 3 & 2 & - & 1 \\
21 bis 25 Jahre & 3 & 7,3 & 1 & 1 & 1 & - & - \\
26 bis 30 Jahre & 4 & 9,8 & 3 & 1 & - & - & - \\
31 bis 35 Jahre & 1 & 2,4 & 1 & - & - & - & - \\
\hline insgesamt & 41 & 100 & 16 & 10 & 8 & 1 & 6 \\
\hline \\
Rundungsdifferenzen sind möglich. \\
a Davon ein Abgeordneter auch 2009 bis 2014 im Europäischen Parlament. \\
Quelle: Vgl. Tabelle 1.
\end{tabular}


abnehmenden Bereitschaft in den Parteien, ältere Abgeordnete noch für die Wahlen zum EP zu nominieren, oder in einem (vermutlich) altersmotivierten Verzicht auf eine erneute Kandidatur. Doch bedeutet selbst diese altersbedingte Ablehnung einer erneuten Kandidatur keineswegs auch den Verzicht auf (zumindest ehrenamtliches) parteipolitisches, gesellschaftspolitisches oder europapolitisches Engagement.

\section{Kein „Opa für Europa ..."?}

Nicht allein für die Gruppe der Parlamentarier, die das 60. oder das 65. Lebensjahr Mitte 2009 noch nicht erreicht hatten, bedeutete das Ausscheiden aus dem Europäischen Parlament nicht zugleich das Ende der berufspolitischen Karriere oder des politischen Engagements. Nur für insgesamt etwa 40 Prozent der 41 Abgeordneten konnte entweder ein altersbedingtes Ausscheiden aus der (aktiven) Politik angenommen werden oder ließen sich keine zuverlässigen Angaben über den Verbleib und die Aktivitäten nach dem Verlassen des Parlaments ermitteln. Für die Mehrzahl der Abgeordneten - und dies betrifft alle Altersgruppen - war indes eine Fortsetzung des berufspolitischen Agierens oder zumindest ein ehrenamtliches politisches oder gesellschaftspolitisches Engagement festzustellen.

Im Detail fächerten sich die - überwiegend - politischen Ambitionen auf. Einige EuropaAbgeordnete (drei) strebten ein Bundestagsmandat an, andere konnten, nachdem ihre europapolitischen Ziele gescheitert waren, ersatzweise ein Landtagsmandat erlangen (drei). Ebenfalls mit berufspolitischem Ziel erreichten andere ausgeschiedene Abgeordnete (drei) Regierungs- oder regierungsnahe Funktionen auf Landesebene oder eine hohe Parteifunktion (einer). ${ }^{51}$ Neben ehrenamtlichen Funktionen in den Parteien, in Wohlfahrtseinrichtungen oder im Rundfunkbeirat findet sich eine Fortsetzung des europapolitischen Engagements durch Beratungs- und journalistische Tätigkeiten und schließlich auch in Form lobbyistischer Dienstleistungen.

Nur für wenige Parlamentarier war die Annahme des EP-Mandat nicht auf ein mitteloder langfristiges europapolitisches Engagement ausgerichtet. Von den zwölf MdEP, die 2009 ausschieden und nur eine Wahlperiode (bis zu fünf Jahren) absolvierten (vgl. Tabelle 8), kann für drei oder vier mit einiger Sicherheit angenommen werden, dass das EP für sie nur ein Interim darstellte, bevor sie wieder auf der bundespolitischen Bühne aktiv werden konnten (Cem Özdemir, Sarah Wagenknecht, Frithjof Schmidt, auch Angelika Beer). Anderen gelang ein gewünschter längerfristiger Wechsel vom Landesparlament in das EP nicht (so dem Nachrücker Roland Gewalt, nach 15 Jahren MdL und MdB), oder sie vermochten es nicht, den parlamentarischen Start im EP in der folgenden Wahlperiode fortzusetzen, ohne dass deshalb das berufspolitische Engagement aufgegeben wurde (Tobias Pflüger, Gisela Kallenbach - beide hatten einen aussichtslosen Listenplatz).

Nur für zwei oder drei MdEP, die nur eine Wahlperiode im EP saßen, lassen sich keine berufspolitischen Ambitionen feststellen (Vugar Öger, Kurt Joachim Lauk, eventuell auch Willem Schuth). Ihre Nominierung hatte vermutlich die Intention, bestimmte wirtschaftli-

51 Dennoch dienen die EP-Abgeordneten nur in Ausnahmefällen als Rekrutierungsreserve für Bundes- oder Landesregierungen, was sich bereits bei der Betrachtung der vorzeitig ausgeschiedenen Europa-Abgeordneten gezeigt hatte. Der Wechsel von Helmuth Markov (PDS) als Finanzminister in die Regierung des Landes Brandenburg kann als Ausnahme angesehen werden. 
che und gesellschaftspolitische Einflussgruppen europapolitisch stärker zu repräsentieren. Bei einem weiteren Abgeordneten mag vielleicht auch eine Form politischer Alimentation bei der Nominierung eine Rolle gespielt haben (Milan Horáček), was eine weitere Kandidatur verhinderte.

Was sich anhand dieser zwölf Europa-Abgeordneten so gut wie gar nicht aufzeigen lässt, ist die Nutzung von EP-Mandaten, um altgedienten Parlamentariern / Politikern ein „Ausschleichen“ aus der aktiven Politik zu ermöglichen. Nur zwei hatten zum Zeitpunkt der Konstituierung des 6. EP bereits das 60. Lebensjahr überschritten (Gisela Kallenbach, Vural Öger), beide waren zum Zeitpunkt ihrer Wahl noch keine (langgedienten) Berufspolitiker (und Gisela Kallenbach wollte und konnte ihre parlamentarische Tätigkeit nach dem Ausscheiden aus dem EP 2009 mit einem Landtagsmandat fortsetzen).

Das Europäische Parlament wird inzwischen als Teil des europäischen politischen Mehrebenen-Systems angesehen. Aufgrund seiner politischen Aufwertung durch Kompetenzerweiterungen liegt die Frage nahe, ob es einen ausgeprägten Wechsel der Abgeordneten zwischen nationaler (Bundestag, Landesparlamente) und europäischer Repräsentation gibt. ${ }^{52}$ Auch wenn für einige ausgeschiedene Parlamentarier, der Wechsel in den Bundestag (drei) oder in ein Landesparlament (vier) festgestellt werden konnte, spricht dies jedoch bislang nicht für eine als typisch anzusehende Parlamentarier-Zirkulation im Mehrebenen-System. Das lässt sich auch für die entgegengesetzte Richtung nicht feststellen. Von den 41 ausgeschiedenen Europa-Parlamentariern waren zwölf (29,3 Prozent) vorher Mitglied des Bundestages (sechs) oder eines Landesparlaments (sieben, da ein Abgeordneter auch einige Zeit MdB war). Für vier Abgeordnete war das Bundestagsmandat nur ein kurzfristiges Engagement, das sich nicht einmal über eine Wahlperiode erstreckte (ein bis drei Jahre). Für zwei $\operatorname{MdEP}$ (Beer, Özdemir) lag der politische Handlungsschwerpunkt eindeutig auf der Bundespolitik - sie gehörten dem Bundestag elf beziehungsweise acht Jahre an -, während das Europa-Mandat für sie ganz offensichtlich nur ein Interim darstellte. Für vier Abgeordnete, die zwei und mehr Wahlperioden im EP verbrachten, können vorangehende lange Mandatszeiten in Landtagen (9, 12, 14 und 20 Jahre) festgestellt werden. Zwar scheinen beide Parlamente (EP / Landesparlamente) eine gleichwertige Bedeutung für die Abgeordneten gehabt zu haben (gemessen an der verbrachten Zeit). Doch zeigt sich durchgehend ein „Aufstieg“ vom Landes- zum Europa-Parlament. 53

Als typisch für die mit dem Ende der 6. Wahlperiode aus dem EP ausgeschiedenen Abgeordneten kann vielmehr eine lange Verweildauer in Parlamenten und auch im Europäischen Parlament gelten (zehn und mehr Jahre). Es handelt sich überwiegend um Berufspolitiker, für die die Parlamentstätigkeit den Mittelpunkt ihres politischen Engagements darstellte und die auch nach dem Ausscheiden noch überwiegend (berufs-)politischen oder gesellschaftspolitischen Ambitionen nachgingen.

52 Die Frage kann hier nur beiläufig angesprochen werden, da den Gründen für einen Wechsel vom Bundestag oder einem Landesparlament in das Europäische Parlament hier nicht weiter nachgegangen werden konnte.

53 Die drei Abgeordneten (André Brie, Gisela Kallenbach, Angelika Beer), die nach dem Ausscheiden aus dem EP ein Mandat in einem Landesparlament erlangten, hatten diese Option - ersatzweise - erst angestrebt, nachdem ein Verbleib im EP zwar gewünscht, aber nicht realisiert werden konnte. Hingegen wurden von den drei Abgeordneten, die nach dem Ausscheiden aus dem EP ein Bundestagsmandat erreichten, diese Mandate gezielt angestrebt, auch indem auf eine erneute Kandidatur für das EP verzichtet wurde. 
Auch das böse Wort vom nach Europa geschickten „Opa“ findet - für die mit dem Ende der 6. Wahlperiode 2009 ausgeschiedenen Europa-Abgeordneten - keine Bestätigung, auch nicht bei jenen Abgeordneten, die nur eine Wahlperiode im EP vertreten waren. Das Europäische Parlament wurde von den Parteien so gut wie gar nicht dazu genutzt, langgedienten Politikern einen parlamentarischen „Alters(ruhe)sitz“ zu gewähren. 54

54 Es kann nicht völlig ausgeschlossen werden, dass es nicht auch eine Art „Gefälligkeitsnominierung“ gab, doch hatte kein Abgeordneter, der nur eine Wahlperiode im EP saß, zum Zeitpunkt des Einzugs in das EP bereits das 65. Lebensjahr erreicht. Gegen eine derartige Vergabe von EuropaMandaten spricht aber auch die ausgeprägte Konkurrenz bei der Nominierung für ein Mandat und auch die Anforderung bei der Listenaufstellung, regionale Parteigliederung und gegebenenfalls auch Partei-Strömungen zu berücksichtigen. Die insgesamt dann doch sehr geringe Zahl von erfolgversprechenden Listenplätzen bietet den Parteien nur in geringem Umfang die Chance für „Gefälligkeitsnominierungen“. Diese Einschätzung wird jedoch nicht dadurch grundsätzlich ins Gegenteil verkehrt, dass für die 7. Wahlperiode Monika Hohlmeier (47, CSU), Reinhard Bütikofer (56, Die Grünen) und Lothar Bisky (67, Die Linke) - Altersangabe jeweils für den Zeitpunkt der Konstituierung des Europäischen Parlaments - erfolgreich nominiert wurden.

\title{
Das novellierte Gesetz über die Zusammenarbeit von Bundesregierung und Deutschem Bundestag in Angelegenheiten der Europäischen Union (EUZBBG)
}

\author{
Hinrich Schröder*
}

Am 13. Juli 2013 trat das neu gefasste Gesetz über die Zusammenarbeit von Bundesregierung und Deutschem Bundestag in Angelegenheiten der Europäischen Union (EUZBBG) ${ }^{1}$ in Kraft. Wie sein Vorgänger ${ }^{2}$ konkretisiert es die in Art. 23 Abs. 2 und 3 des Grundgesetzes (GG) statuierten Unterrichtungs- und Stellungnahmerechte des Bundestages. Es soll einfachgesetzlich sicherstellen, dass der Bundestag von der Bundesregierung über alle Angelegenheiten der Europäischen Union umfassend und zum frühestmöglichen Zeitpunkt unterrichtet wird und sein Recht zur Stellungnahme effektiv wahrnehmen kann.

Eine Änderung des Gesetzes war erforderlich geworden, nachdem das Bundesverfassungsgericht (BVerfG) in seinem Urteil vom 19. Juni $2012^{3}$ die Unterrichtungsrechte des Bundestages nach Art. 23 Abs. 2 GG weiter ausgelegt hatte, als sie vom bisherigen EUZBBG abgebildet waren. Dem Urteil vorausgegangen war eine - nach Inkrafttreten des EUZBBG

* Der Autor ist Referent im Referat EU-Grundsatzangelegenheiten, Fragen der Wirtschafts- und Währungsunion der Verwaltung des Deutschen Bundestages. Der Beitrag gibt ausschließlich seine persönliche Auffassung wieder.

1 BGBl. 2013 I, S. 2170.

2 Vgl. zum EUZBBG a.F. Hinrich Schröder, Mitwirkung des Bundestages in EU-Angelegenheiten nach dem EUZBBG in der Praxis - ein Kurzkommentar, in: ZParl, 43. Jg. (2012), H. 2, S. 250 $-277$.

3 BVerfG, 2 BvE 4/11 vom 19. Juni 2012; BVerfGE 131, S. 152. 